\title{
Development of an embryo transfer model to study uterine contributions to pregnancy in vivo in mice
}

\author{
Meaghan J Griffiths, Lauren R Alesi, Amy L Winship $\mathbb{B D}^{*}$ and Karla J Hutt
}

Department of Anatomy and Developmental Biology, Development and Stem Cells Program, Biomedicine Discovery Institute, Monash University, Clayton, Victoria, Australia

Correspondence should be addressed to A L Winship or K J Hutt: amy.winship@monash.edu or karla.hutt@monash.edu

*(A L Winship and KJ Hutt contributed equally to this research)

\section{Graphical abstract}

A mouse model to study uterine specific contributions to pregnancy.

Maternal environmental exposures can exert impacts on the ability of the uterus to sustain healthy pregnancy. To establish an in vivo model to study this, we designed an ovariectomized mouse embryo transfer model. The rationale being future studies could expose recipient female mice to variables such as altered diet, drug, temperature, air, or activity exposure among others to define their impacts on the uterine contribution to pregnancy. Ovariectomy ensures the extent of the variable is limited to exploring outcomes on uterine but not ovarian function. Embryo transfer from healthy, unexposed donor mice guarantees that any impacts of the variable are attributed to the maternal uterine but not the embryonic state. Pregnancy outcomes including pregnancy success (number of implantation sites) and viability (number of viable vs resorbing implantation sites) can be investigated. Numerous functional outcomes can be assessed, including developmental competence encompassing decidual, placental, fetal, and vascular morphology and/or function (e.g. measured using Doppler ultrasound, comparisons of fetal growth, or molecular or histological characterization of the decidua, placenta, and fetal tissues).

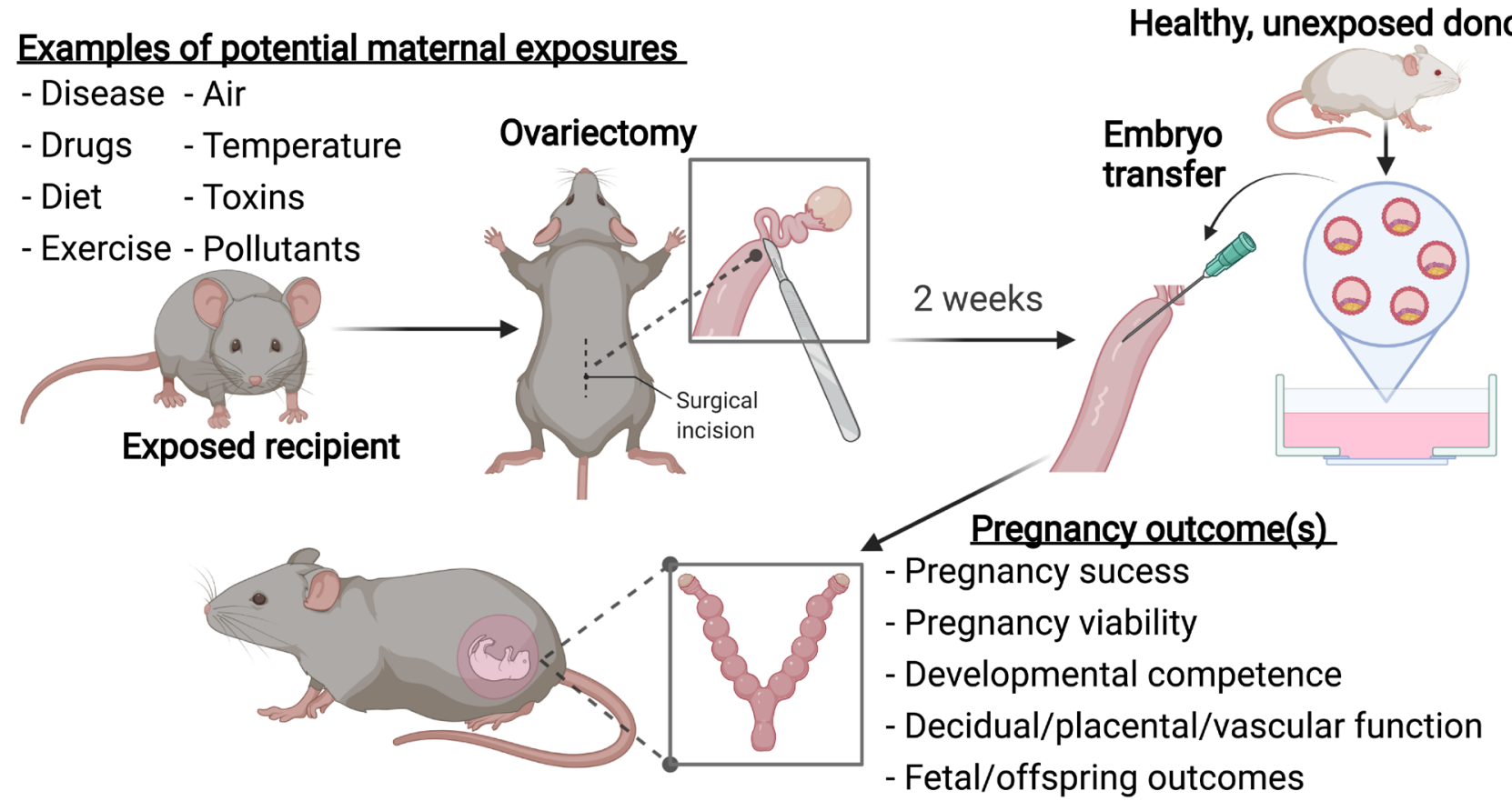

(c) 2022 The authors Published by Bioscientifica Ltd

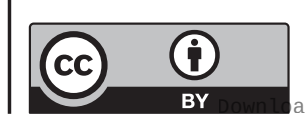
Attribution 4.0 International License. 


\section{Lay summary}

Many pregnancy complications occur because of problems in the womb (uterus), specifically the womb lining. There is a close relationship between the hormone function of the ovaries and the uterus and distinguishing between the way they both impact pregnancy success is difficult in existing studies using animals. Here, we developed a new animal model to utilize in addressing these gaps in our understanding of pregnancy.

Key Words: - endometrial receptivity $\quad$ pregnancy $\quad$ ovariectomy $\quad$ embryo transfer infertility

Reproduction and Fertility (2022) 2 11-19

\section{Introduction}

Assisted reproductive technologies have rapidly changed the landscape of family planning. However, clinical pregnancy rates following in vitro fertilization or intracytoplasmic sperm injection have stagnated in recent years (Norwitz et al. 2001, Kupka et al. 2014, Ledee et al. 2016), indicating many morphologically good-quality embryos fail to implant and establish a pregnancy. This suggests other barriers, including uterine deficiencies, contribute to the limited pregnancy success rates observed clinically (Richter et al. 2006, Ruiz-Alonso et al. 2013, 2014, Coughlan et al. 2014). While informative, human in vitro culture models of endometrial receptivity and early implantation processes, including decidualization (Fitzgerald et al. 2019, 2021), do not allow for the complete recapitulation of the dynamic processes involved in pregnancy establishment. As such, existing in vivo models make it difficult, if not impossible, to study uterine specific contributions to pregnancy success, as distinct from those arising from ovarian impacts.

For the maternal environment to be optimally prepared for pregnancy, the endometrium must be receptive to a viable embryo (Evans et al. 2016). Once implantation occurs successfully, the maternal stroma terminally differentiates into decidual cells that secrete progesterone to support the pregnancy until the placenta develops (Evans et al. 2016). Deficits at any of these early stages can result in pregnancy loss (Wu et al. 2018, Yang et al. 2019). In recent years, the endometrial receptivity array was developed to assist women with a delayed or shifted window of receptivity to tailor the timing of embryo transfer (ET) to match any individual woman's optimal receptivity window (Diaz-Gimeno et al. 2011, Patel et al. 2019). However, the field still lacks an understanding of the uterine drivers of implantation failure and early pregnancy loss and the development of pregnancy complications that manifest after successful implantation.
Female reproductive health and function are reliant on a delicate balance of ovarian hormones that regulate the uterine menstrual cycle. The ovarian granulosa and theca cells produce and secrete estrogen to regulate the menstrual cycle and ovarian follicle development. When ovarian hormones are not produced, the uterus can still respond to exogenous hormone replacement. As an example, young female cancer patients who have undergone sterilizing treatment can successfully transition through puberty with hormone replacement therapy (Critchley et al. 2002, Critchley \& Wallace 2005, Sudour et al. 2010).

The impact of exogenous insults on the ovary's endocrine function and fertility are well documented with well-established models to demonstrate ovarian follicle depletion following cancer therapies and exposure to environmental toxins (Winship et al. 2018). However, oversights and inherent flaws in the study design of these models can mean that any specific uterine damage inflicted by exogenous insults cannot be accurately differentiated from secondary effects arising from ovarian damage. To overcome this issue, ovariectomized mice are commonly used in studies investigating specific insults to the endometrium (Greaves et al. 2014, Fullerton et al. 2017).

For pregnancy studies, conditional knockout mice using the Cre-lox recombination system are frequently employed, where a tissue-specific Cre-recombinase excises a gene locus flanked by loxP sites to manipulate gene expression in a time- and tissue-specific manner (Feil et al. 2009). Driven by progesterone receptor expression, the PR-Cre (Daikoku et al. 2008, Fullerton et al. 2017) limits loss-of-function of the target gene to tissues and cells expressing progesterone receptor. An important limitation to this system when considering uterine specific deficits is that the expression profile of progesterone receptor extends beyond the uterus, with other tissues including oviduct, ovary, mammary gland, and the pituitary gland (Daikoku et al. 2008) also expressing progesterone receptor. This system also does not allow for the uterine specific impacts

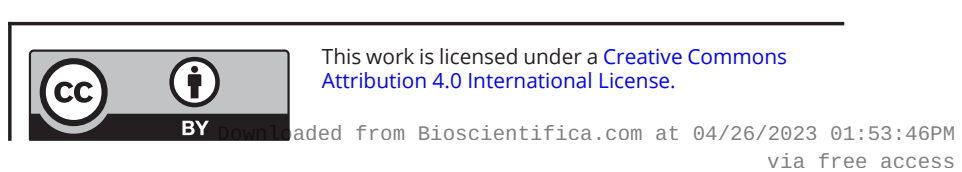


of exogenous insults to be studied, highlighting the need for an animal model to specifically investigate exogenous factors on uterine function and allow distinction from ovarian and endocrine influence. ETs where blastocysts are collected from donor mice that remain unexposed to an exogenous treatment provide a well-controlled model for studying uterine specific defects in pregnancy.

Here, we used the common inbred C57BL/6J strain, as well as an F1 cross of C57BL6J and BALB/c, and the outbred strain Swiss to optimize an ET model for studying uterine contributions to pregnancy complications. We aimed to define implantation rates following ET across strains. We determined the extent of developmental progression following ET and exogenous hormone supply in each strain and also assessed uterine artery blood flow in pregnancy across each strain.

\section{Materials and methods}

\section{Animals}

Animals were housed in temperature-controlled high barrier facilities (Monash University Experimental Animal Facility), with food and water available ad libitum under a $12 \mathrm{~h}$ light:12 h darkness cycle. All procedures were approved by the Monash University Animal Ethics Committee and performed in accordance with the NHMRC Australian Code of Practice for the Care and Use and Animals. Surgical procedures for this study were completed by the Monash Animal Research Platform Reproductive Services Team.

\section{Ovariectomy of embryo transfer recipients}

Based on a previously published methodology (Kondoh et al. 2009), 6-week-old female C57BL/6J, Asmu:Swiss, or C57BL/6JxBALB/cJAsmu(F1) were ovariectomized. Females were anaesthetized by isoflurane inhalation (1-5\%, $\mathrm{v} / \mathrm{v}$ in oxygen). Analgesic carprofen was administered subcutaneously (5 $\mathrm{mg} / \mathrm{kg}$ in saline) and eye lubricant was applied. A small region was shaved on the animals back where a paralumbar skin incision was then made. Blunt dissection was used to separate the skin from the underlying muscle before small incisions were made through the peritoneal muscle wall just under the kidney on each side. The ovaries and oviducts were removed, the peritoneal muscle wall incisions were closed with a double suture knot (Sofsilk Silk sutures GS-832, Covidien), and bupivacaine analgesic was applied topically $(0.05 \%$ in saline). Skin incisions were closed with Michel clips
(Daniels, NS-000242). Female mice were then rested for 2 weeks to allow endogenous hormones to subside.

\section{Embryo transfer donors}

Adult female BALB/cJAsmu were superovulated via injection with PMSG (Folligon, 5 IU) on day (D) 1, then hCG (Chorulon, 5IU) on D3, and then mated with a $\mathrm{BALB} / \mathrm{cJAsmu}$ stud overnight. Those that had plugged the following morning were then humanely culled by cervical dislocation on D6 (gestational day 2.5) and their uterine horns and oviducts flushed for blastocysts. Blastocysts were cultured overnight in M2 media before transfer to recipient animals the following day.

\section{Embryo transfers to recipients}

On D0, females received 100 ng estradiol (Sigma E8875) and then $2 \mathrm{mg}$ progesterone (Sigma P0130) on D2 in sesame oil. On D3, ETs were performed. Females were anaesthetized via isoflurane inhalation (1-5\%, v/v in oxygen) and a small region was shaved on the animals back. Analgesic carprofen (5 $\mathrm{mg} / \mathrm{kg}$ in saline) was administered subcutaneously and a paralumbar incision made through the s.c. layer. Each uterine horn was exposed at the oviduct end via a small incision into the peritoneal wall for transfer of five blastocysts per horn. Peritoneal incisions were closed using double suture knot (Sofsilk Silk sutures GS-832, Covidien) and then bupivacaine (0.05\% in saline) was applied topically. At the time of ET, females also received $2 \mathrm{mg}$ progesterone and $25 \mathrm{ng}$ estradiol subcutaneously. Progesterone ( $2 \mathrm{mg}$ ) was supplemented each day post ET, until collection 10 days post ET. Animals were humanely culled by isoflurane inhalation after conclusion of ultrasound imaging, and then uteri were carefully dissected out and formalin was fixed for further analysis.

\section{Doppler ultrasonography}

At 10 days post ET, recipient females were anaesthetized by isoflurane inhalation (1-5\% v/v in oxygen) and then transferred to a heated imaging platform in supine position. Uterine artery was identified and imaged using the Vevo2100 system (VisualSonics). Three consecutive waveform measurements were taken from three separate ultrasound recordings of the uterine artery. Measurements included peak systolic velocity (PSV), end diastolic velocity (EDV), and velocity time interval (VTI). Calculations of pulsatility and resistive indices were then calculated as follows: $\mathrm{PI}=(\mathrm{PSV}-\mathrm{EDV}) / \mathrm{VTI}$ and $\mathrm{RI}=(\mathrm{PSV}-\mathrm{EDV}) / \mathrm{PSV}$. 


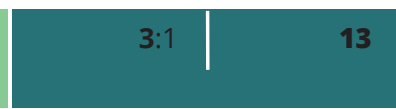

\section{Histological analysis}

Whole implantation sites, dissected embryos, or placentas were formalin fixed for $24 \mathrm{~h}$ and then processed and embedded in paraffin and sectioned at $5 \mu \mathrm{m}$. Sections were dewaxed in histolene twice and rehydrated through graded ethanols (100-70\%) and distilled water prior to relevant staining procedure outlined below. For dehydration and clearing, sections were rapidly moved through graded ethanols (70-100\%) and cleared in histolene twice before mounting with DPX and glass cover slips.

\section{Hematoxylin and eosin}

Whole implantation sites, dissected embryos, or placentas slides were submerged in hematoxylin for $10 \mathrm{~min}$, rinsed under running distilled water until clear before being immersion in lithium carbonate for $30 \mathrm{~s}$, and rinsed again prior to dip in acid alcohol and finally eosin for $2 \mathrm{~min}$.

\section{Periodic acid Schiff (PAS)}

Placental sections were placed in periodic acid (Amber Scientific, ChemSupply, Australia) for $10 \mathrm{~min}$, rinsed thoroughly with water for 2 min, placed in Schiff's reagent (Amber Scientific) for $15 \mathrm{~min}$, and rinsed in water again. Sections were then counterstained with hematoxylin only (as described above).

\section{Statistics}

Statistical analysis was performed using Graphpad Prism 9. Data normality was assessed by Shapiro-Wilk test. Significance was assessed using one-way ANOVA with Tukey's multiple comparison test for parametric data or Kruskal-Wallis test with Dunn's multiple comparisons test for non-parametric data. A $P$-value of $<0.05$ was considered significant.

\section{Results}

To enable distinction between uterine and ovarian contributions to pregnancy success, mice were ovariectomized, hormone primed prior to ET, and whole implantation sites, embryos, and placentas collected 10 days post ET (Fig. 1A). All ET recipient strains C67BL/6J, Swiss, and C56BL/6xBALB/c (F1) received transfer of 8-10 $\mathrm{BALB} / \mathrm{c}$ donor embryos. C57BL/6J were chosen as they are an extremely commonly used strain as WT and for generating knockout colonies. Swiss:Asmu is an outbred
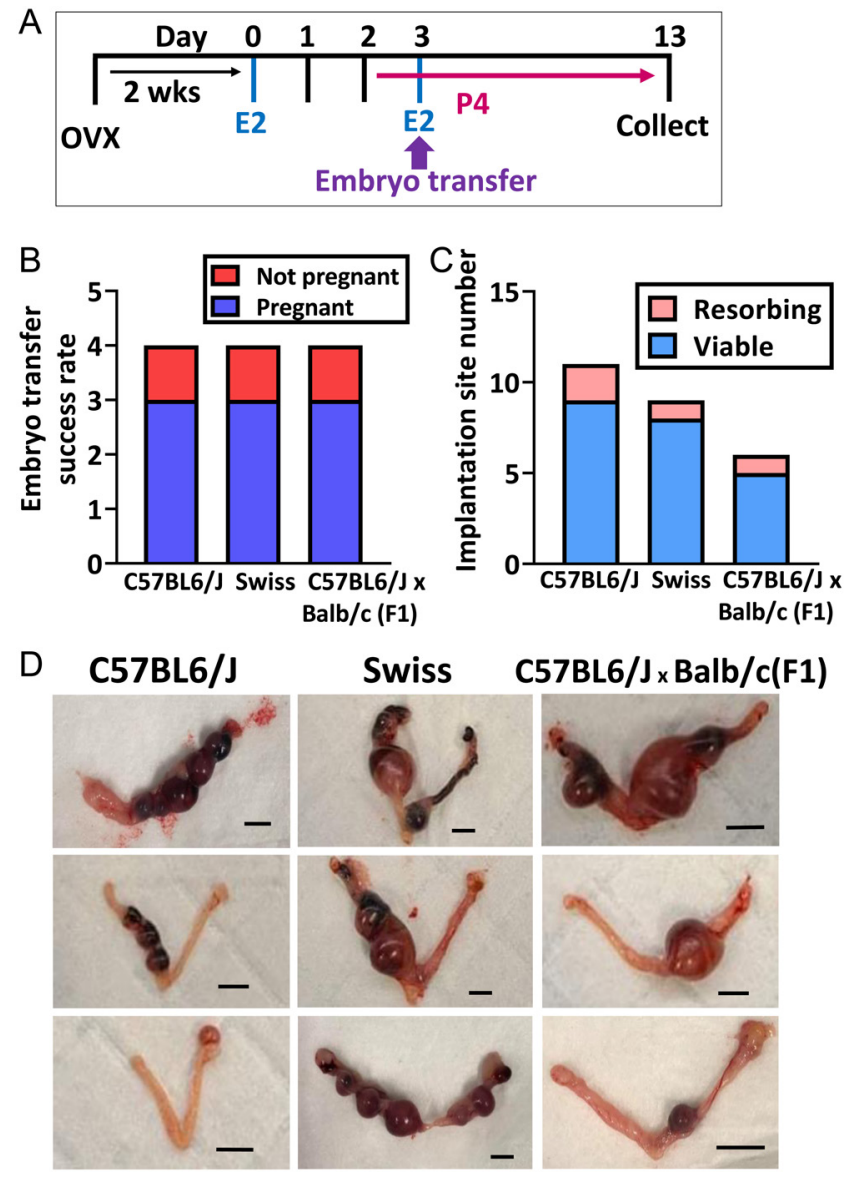

Figure 1 Optimizing an embryo transfer model for studying uterine specific contributions to pregnancy. (A) Young adult female mice were ovariectomized and rested for 2 weeks before hormone priming with estradiol (D0) and progesterone (D2) before embryo transfer surgery on D3. Pregnancy was supported with estradiol ( $25 \mathrm{ng}$ ) at the time of embryo transfer and daily s.c. injections of progesterone $(1 \mathrm{mg})$ from D3 until time of tissue collection. Implantation sites were harvested 10 days post embryo transfer. (B) The embryo transfer success rate shows three out of four mice of each strain were pregnant at D13. (C) Implantation rates per animal were similar. (D) Panels show images of each pregnant uterus from each strain and the variety in developmental stage of each implantation site. Scale bars $5 \mathrm{~mm}$. Data are shown as mean \pm S.E.M.; one-way ANOVA with Tukey's multiple comparisons test; $n=4$ /group.

strain useful for determining if there was any difference in the success of our model with more genetic diversity bred into the colony. Finally, we included a mixed background cross of the commonly available C57BL/6J and BALB/c.

Swiss recipient mice had the greatest body weight at the time of collection, despite consistent ages across the recipients (Supplementary Fig. 1, see section on supplementary materials given at the end of this article). ET success rate was $75 \%$ for each strain (Fig. 1B). C57BL/6J and Swiss recipient mice had similar numbers of implanted embryos and of the number that were viable was also similar (Fig. 1C and Table 1). C57BL/6J were the least

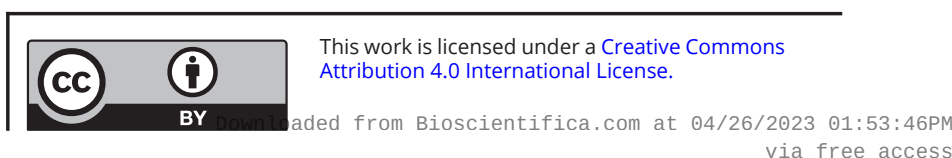


Table 1 Embryo transfer success. The number of viable implanted embryos, and resorbing non-viable implantation sites for each strain at 10 days post embryo transfer. Data are represented as mean \pm S.E.M.

\begin{tabular}{l}
\hline Strain \\
\hline C57BL6/J \\
Asmu:Swiss \\
C57BL6/J $\times$ Balb/C(F1)
\end{tabular}

\begin{tabular}{c}
\hline Transferred \\
\hline 10 \\
10 \\
10
\end{tabular}

\begin{tabular}{c}
\hline Implanted \\
\hline $3.33 \pm 1.20$ \\
$3.00 \pm 1.00$ \\
$2.00 \pm 1.00$ \\
\hline
\end{tabular}

\begin{tabular}{c}
\hline Viable \\
\hline $2.67 \pm 1.53$ \\
$2.67 \pm 2.08$ \\
$1.67 \pm 1.16$ \\
\hline
\end{tabular}

\begin{tabular}{c}
\hline Resorbing \\
\hline $0.67 \pm 0.57$ \\
$0.33 \pm 0.57$ \\
$0.33 \pm 0.57$ \\
\hline
\end{tabular}

developmentally advanced (Fig. 1D) and had the most resorbing sites (Fig. 1D and Table 1). The outbred Swiss strain were much more developmentally advanced (Fig. 1D) compared to the C67BL/6J females. The F1 females had the smallest number of implantation sites (total implanted and viable implantation sites $1.67 \pm 1.16$ ) (Fig. 1D); however, their developmental progression was similar to that of the Swiss females.

Due to the advanced developmental progression of the Swiss and F1 strains, embryos and placentas were dissected from each of these strains for morphological and histological assessment (Fig. 2A). This was not possible for C57BL/6J implantation sites because they were at an earlier developmental stage. Hence, the placenta and embryo were not yet fully formed.

Developmentally, embryos that developed in Swiss mothers were less advanced compared to embryos from the F1 strain. Figure 2B displays the developing brain structures of an embryo from a Swiss mother, compared to the more advanced $\mathrm{F} 1$ strain embryo which displays more developed brain structures and abdominal organs, with the liver observable (Fig. 2B). There were no obvious differences in gross morphology of the placentas between maternal strains with clear distinction between the labyrinth and spongiotrophoblast layers in both strains visualized through PAS staining (Fig. 2C).
$\underline{A}$

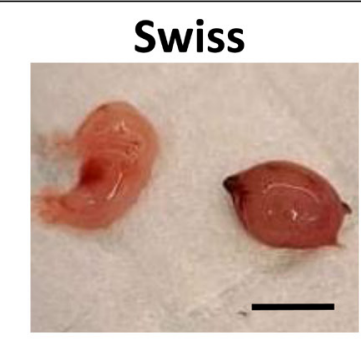

B
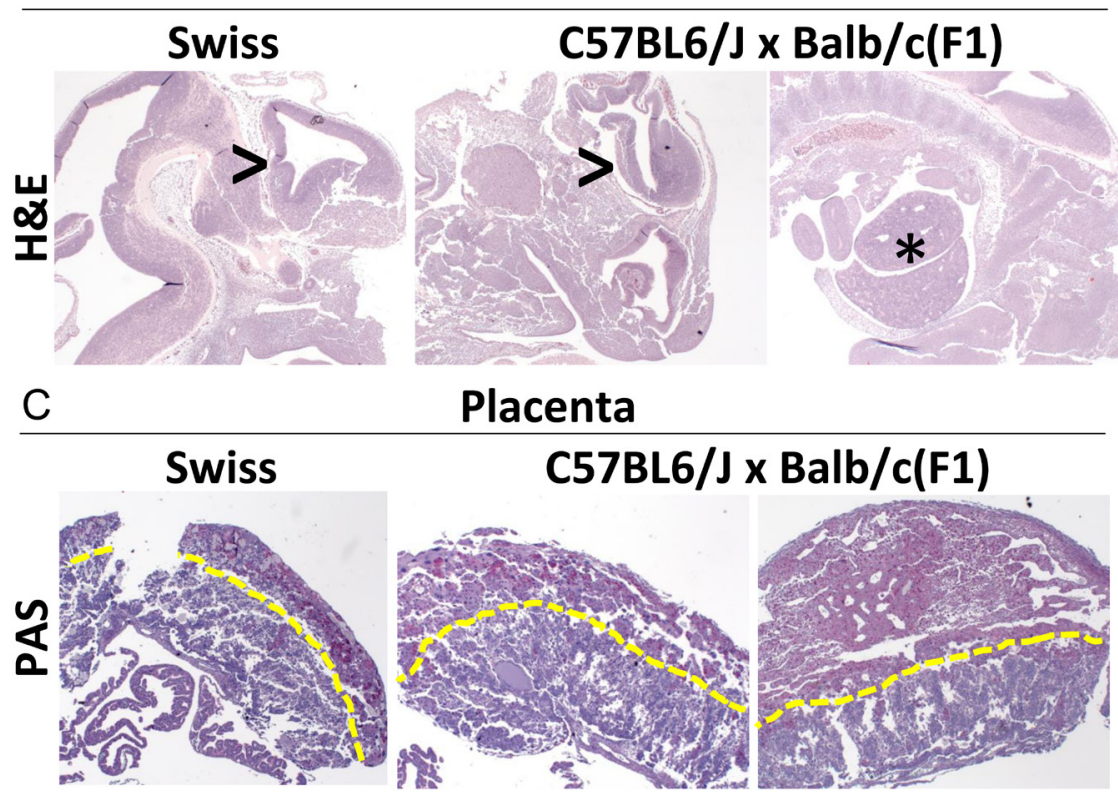

https://raf.bioscientifica.com https://doi.org/10.1530/RAF-21-0087

\section{Macroscopic}
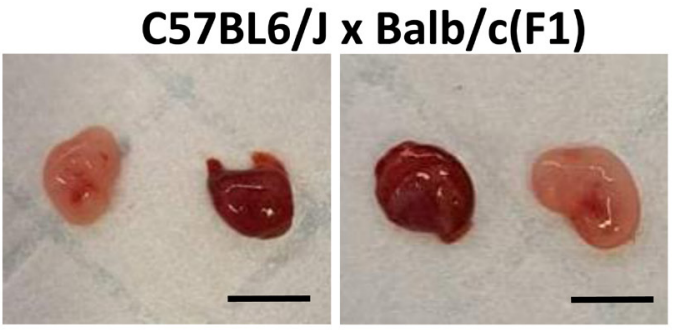

Fetus i.
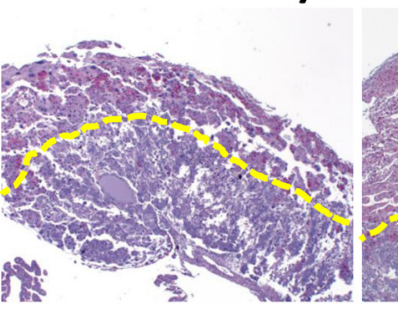

(C) 2022 The authors Published by Bioscientifica Ltd
Figure 2 Histological features of fetal and placental tissues from Swiss and F1 mothers. (A) Macroscopic images of dissected implantation sites from Swiss and F1 females, which had the most developmentally advanced implantation sites. (B) Representative cross sections of fetal tissues. Forebrain structures (>) appear less developed in offspring from Swiss mothers compared to the F1 strain. Additionally, development of other organs including the liver (*) are apparent in offspring from F1 mothers. (C) From representative photomicrographs of PAS staining, there were no apparent differences in gross placental morphology between strains. Yellow dotted line separates labyrinth and spongiotrophoblast sections of the placenta. Images taken at $10 \times$ objective.

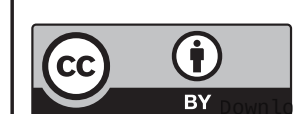

This work is licensed under a Creative Commons Attribution 4.0 International License. 
A
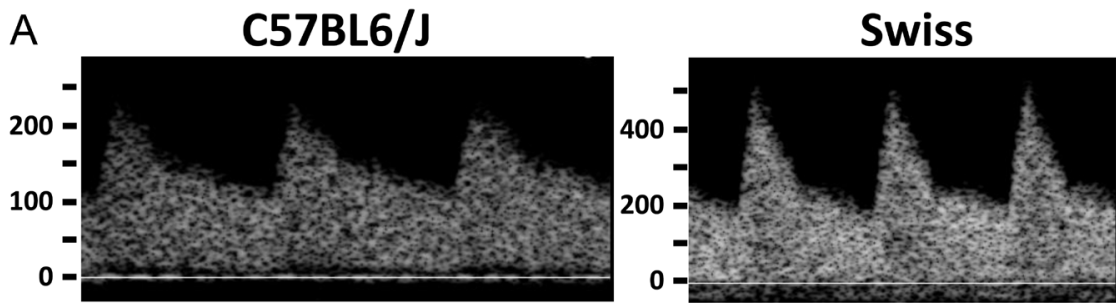

C57BL6/J x Balb/c(F1)
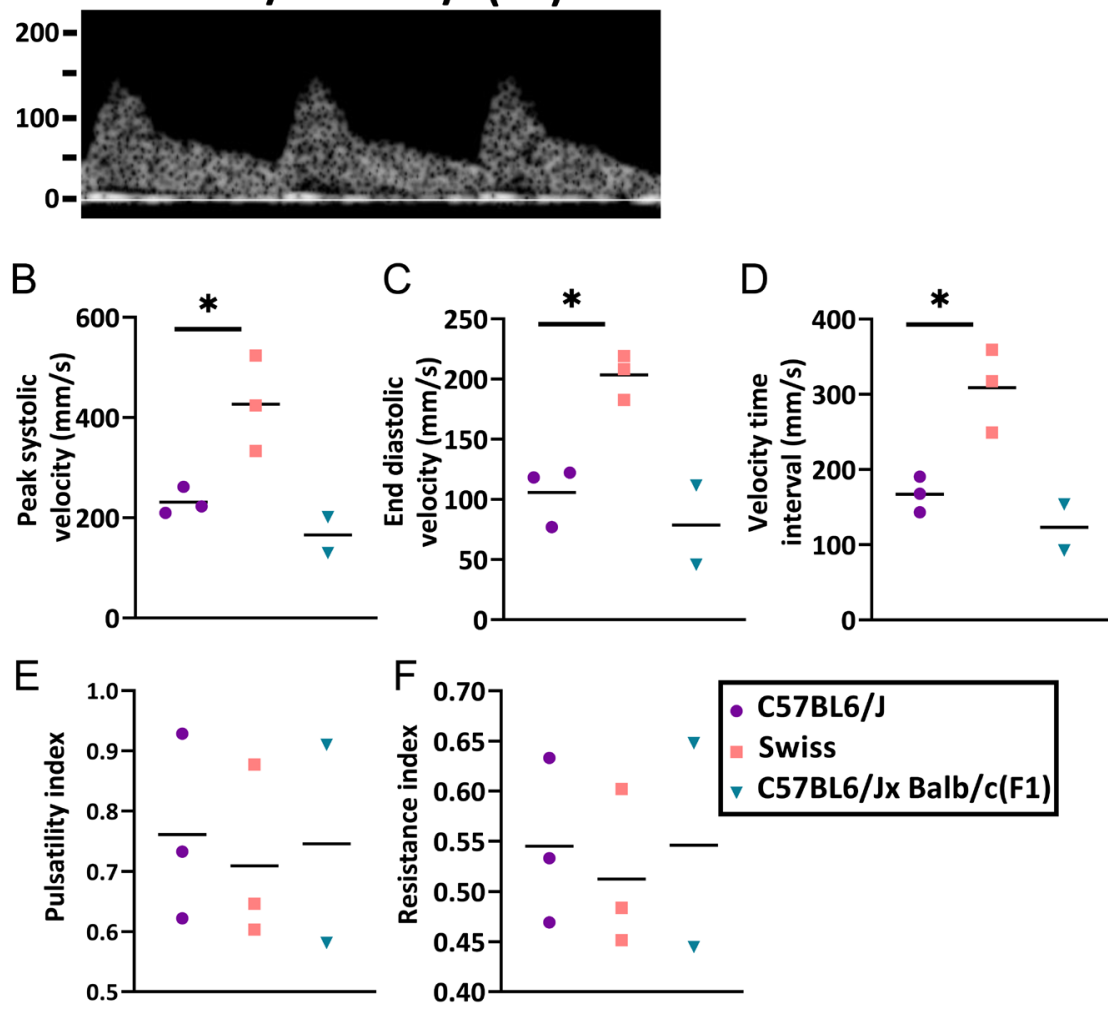

Figure 3 Uterine artery blood flow is most readily detectable by Doppler ultrasound in the outbred Swiss strain. (A) Representative images of uterine artery waveforms. Outbred Swiss have greater peak systolic velocity (B), end diastolic velocity (C), and velocity time interval (D). No changes in pulsatility (E) or resistance (F) indices. Data are shown as mean \pm S.E.M.; unpaired $t$-test between C57BL6J and Swiss groups only for (B-F); $n=2-4$ /group.

Adaptations to maternal blood flow during early pregnancy are critical for ongoing pregnancy success. Doppler ultrasonography waveforms are greatly informative, both in their shape and velocity measurements (Clark et al. 2018). Clinically, uterine artery Doppler is used to assess the development of pregnancy complications (Nakatsuka et al. 2003, Papageorghiou \& Leslie 2007, Ridder et al. 2019). Measurements of uterine artery blood flow taken with Doppler ultrasonography revealed significantly higher peak systolic velocity, end diastolic velocity, and velocity time interval in outbred Swiss mice compared to C57BL/6J mice (Fig. 3). While the F1 strain also appeared to have lower velocity blood flow, statistical analysis was not possible due to the small sample size. Pulsatility and resistance indexes were similar for all strains, indicating changes to the velocity of the system did not appear to influence the function of the uterine artery (Fig. 3).

Table 2 Advantages and weaknesses of each strain for use in embryo transfer studies.

\begin{tabular}{ll}
\hline Strain & \multicolumn{1}{l}{ Advantages } \\
\hline C57BL6/J & - Multiple implantation sites \\
Swiss & - Ideal for studies of early implantation \\
C57BL6/J $\times$ Balb/c(F1) & - High-velocity uterine blood flow \\
& - Low risk of interventions post surgery \\
& Ideal for decidualization and placentation studies \\
\hline
\end{tabular}

\section{Limitations}

- Slower developmental progression

- May require clip repairing or culling post surgery

- May require clip repairing or culling post surgery

- Difficult to detect blood flow https://raf.bioscientifica.com

https://doi.org/10.1530/RAF-21-0087 (c) 2022 The authors Published by Bioscientifica Ltd

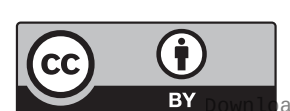

This work is licensed under a Creative Commons Attribution 4.0 International License. 


\section{Discussion}

Here, we present a strategy for deciphering damage to the uterus from ovarian or endocrine influence in a mouse model. By ovariectomizing mice and then providing hormone support exogenously alongside ET from healthy donor mice, specific insults to the uterus and their impacts on pregnancy can be determined. We compared this model in three commonly available and widely utilized strains including the inbred C57BL/6J strain, outbred Asmu:Swiss, and the C57BL/6JxBALB/ $\mathrm{c}(\mathrm{F} 1)$ strain. Depending on the research interests and biological questions being asked by researchers, each of these strains have their advantages and disadvantages (Table 2); however, the key finding of this study advocates that ET success is possible in each of the recipient strains used.

For studies interested in the peri-implantation and early implantation periods (Table 2), C57BL/6J may be most useful as they can generate several implantation sites per animal and are readily available. This is reassuring knowledge as an abundance of genetically modified mouse models are maintained on a C57BL/6 background (Bryant 2011, Fontaine \& Davis 2016).

Alternatively, for studies focussed instead on decidualization or placentation (Table 2), the Swiss or F1 strain may be more appropriate. Swiss have many advantages including being an outbred strain which introduces immune system and genetic diversity (Martin et al. 2017). The Swiss recipient females also had easily detectable and high velocity uterine artery blood flow as determined by Doppler ultrasound making them great candidates for studies focussed on vascular remodeling in pregnancy directly resulting from uterine defects.

In contrast, the F1 strain had much smaller litters, and the uterine artery blood flow was difficult to detect. However, an advantage of the F1 strain is their demeanor and physical condition; they were easy to handle and tolerated surgeries well, with no interventions required to manage the surgical clips. Whereas, both Swiss and C57BL/6J recipients required clip repairs and culling post surgery due to issues with surgical clips. The F1 strain also make good mothers as they are less likely to cannibalize their litters, which the C56BL/6J mothers are known for (Weber et al. 2013, Brajon et al. 2021).

Until now, it has been difficult to differentiate damage to the uterus from damage to other reproductive organs, even in the commonly used PR Cre-lox system. However, a key limitation to the PR-Cre model lies in the fact PR is expressed in many tissues outside the uterus.
While useful for conditional genetic deletions, the PR-Cre system suffers the same shortfalls in limiting researchers' ability to decipher uterine damage from damage to other organs or tissues that may be having downstream impacts on the uterus.

A limitation in our model for consideration is the influence of seminal fluid on the uterine microenvironment and its impact on pregnancy success (Schjenken \& Robertson 2015, 2020). Mating recipient females with vasectomized males is a well-established protocol for inducing pseudo-pregnancy in female mice (Barrette et al. 2012, Rhee et al. 2016). The literature suggests that seminal plasma exposure is important for successful pregnancy establishment (Schjenken \& Robertson 2015) due to its involvement in generating a maternal immune response to promote tolerance to an implanting blastocyst. Despite this, ET was successful in $75 \%$ of females. In the future, introducing a vasectomized mating to this model could further improve the pregnancy and implantation success rates.

The use of non-surgical ET (NSET) devices is a wellestablished method for performing ET surgeries noninvasively. While this device offers a great non-surgical alternative, NSET devices are expensive to purchase commercially and less efficient when made in house. However, the procedure of a NSET could be completed by any trained researcher, while the surgical alterative requires expertise that may not be available to all researchers or institutes.

The findings from this study provide a robust model for studying the specific impacts to the uterus in response to any exogenous insult. By ovariectomizing to remove the confounding factor of any ovarian damage following an external insult and subsequently providing healthy donor embryos from unexposed mice, the specific outcomes on the uterus and on pregnancy following can be elucidated.

\section{Conclusions}

Here, a novel model is described that is designed to differentiate uterine from ovarian influences on pregnancy. Specific impacts to the uterus in response to exposure to exogenous factors can be interrogated using this model, independent of ovarian and endocrine impacts. Examples of exogenous factors include maternal diets, pesticide or herbicide exposure, environmental conditions like smoke or hypoxia exposure, or genotoxic treatments like cancer therapies. This model can also be expanded to 
investigate exposure to potentially beneficial factors to pregnancy success, with the intention of furthering our understanding of the uterine specific factors that help or hinder pregnancy success.

\section{Supplementary materials}

This is linked to the online version of the paper at https://doi.org/10.1530/ RAF-21-0087.

\section{Declaration of interest}

The authors declare that there is no conflict of interest that could be perceived as prejudicing the impartiality of the research reported.

\section{Funding}

This work was made possible through Victorian State Government Operational Infrastructure Support and Australian Government NHMRC IRIISS. M J G and L R A are supported by an Australian Government Research Training Program Scholarship and L R A a Monash Graduate Excellence Scholarship. This work was supported by generous funding support of a Monash University Faculty of Medicine, Nursing \& Health Sciences Platform Access Grant to A L W (PAG18-0343). A L W and KJ H are supported by funding from the Australian Research Council; A L W DE21010037 and KJ H - FT190100265.

\section{Author contribution statement}

M J G, A L W, KJ H conceived and designed the study. M J G, L R A performed experiments. M J G analysed data and all authors interpreted the data. M J $G$ and $A L W$ wrote the manuscript. All authors edited the manuscript. $A L$ W or KJ H contributed equally to this work.

\section{Acknowledgements}

The authors would like to acknowledge the technical support of the Monash Animal Research Platform, in particular the Reproductive Services Team, as well as the Monash Histology Platform and the Monash Micro Imaging facility. Graphical Abstract was created using BioRender.

\section{References}

Barrette VF, Adams MA \& Croy BA 2012 Endometrial decidualization does not trigger the blood pressure decline of normal early pregnancy in mice. Biology of Reproduction 86 66. (https://doi.org/10.1095/ biolreprod.111.096958)

Brajon S, Morello GM, Capas-Peneda S, Hultgren J, Gilbert C \& Olsson A 2021 All the pups we cannot see: cannibalism masks perinatal death in laboratory mouse breeding but infanticide is rare. Animals 11 2327. (https://doi.org/10.3390/ani11082327)

Bryant CD 2011 The blessings and curses of C57BL/6 substrains in mouse genetic studies. Annals of the New York Academy of Sciences 1245 31-33. (https://doi.org/10.1111/j.1749-6632.2011.06325.x)
Clark AR, James JL, Stevenson GN \& Collins SL 2018 Understanding abnormal uterine artery Doppler waveforms: a novel computational model to explore potential causes within the utero-placental vasculature. Placenta 66 74-81. (https://doi.org/10.1016/j. placenta.2018.05.001)

Coughlan C, Ledger W, Wang Q, Liu F, Demirol A, Gurgan T, Cutting R, Ong K, Sallam H \& Li TC 2014 Recurrent implantation failure: definition and management. Reproductive Biomedicine Online 28 14-38. (https://doi.org/10.1016/j.rbmo.2013.08.011)

Critchley HO \& Wallace WH 2005 Impact of cancer treatment on uterine function. JNCI Monographs 2005 64-68. (https://doi. org/10.1093/jncimonographs/lgi022)

Critchley HO, Bath LE \& Wallace WH 2002 Radiation damage to the uterus - review of the effects of treatment of childhood cancer. Human Fertility 5 61-66. (https://doi.org/10.1080/1464727022000198942)

Daikoku T, Hirota Y, Tranguch S, Joshi AR, DeMayo FJ, Lydon JP, Ellenson LH \& Dey SK 2008 Conditional loss of uterine Pten unfailingly and rapidly induces endometrial cancer in mice. Cancer Research 68 5619-5627. (https://doi.org/10.1158/0008-5472.CAN-081274)

Diaz-Gimeno P, Horcajadas JA, Martinez-Conejero JA, Esteban FJ, Alama P, Pellicer A \& Simon C 2011 A genomic diagnostic tool for human endometrial receptivity based on the transcriptomic signature. Fertility and Sterility 95 50-60, 60.e1. (https://doi.org/10.1016/j. fertnstert.2010.04.063)

Evans J, Salamonsen LA, Winship A, Menkhorst E, Nie G, Gargett CE \& Dimitriadis E 2016 Fertile ground: human endometrial programming and lessons in health and disease. Nature Reviews: Endocrinology 12 654-667. (https://doi.org/10.1038/ nrendo.2016.116)

Feil S, Valtcheva N \& Feil R 2009 Inducible Cre mice. Methods in Molecular Biology 530 343-363. (https://doi.org/10.1007/978-1-59745471-1_18)

Fitzgerald HC, Dhakal P, Behura SK, Schust DJ \& Spencer TE 2019 Self-renewing endometrial epithelial organoids of the human uterus. PNAS 116 23132-23142. (https://doi.org/10.1073/pnas.1915389116)

Fitzgerald HC, Schust DJ \& Spencer TE 2021 In vitro models of the human endometrium: evolution and application for women's health Biology of Reproduction 104 282-293. (https://doi.org/10.1093/biolre/ ioaa183)

Fontaine DA \& Davis DB 2016 Attention to background strain is essential for metabolic research: C57BL/6 and the international knockout mouse consortium. Diabetes 65 25-33. (https://doi. org/10.2337/db15-0982)

Fullerton Jr PT, Monsivais D, Kommagani R \& Matzuk MM 2017 Follistatin is critical for mouse uterine receptivity and decidualization. PNAS 114 E4772-E4781. (https://doi.org/10.1073/ pnas.1620903114)

Greaves E, Cousins FL, Murray A, Esnal-Zufiaurre A, Fassbender A, Horne AW \& Saunders PT 2014 A novel mouse model of endometriosis mimics human phenotype and reveals insights into the inflammatory contribution of shed endometrium. American Journal of Pathology 184 1930-1939. (https://doi. org/10.1016/j.ajpath.2014.03.011)

Kondoh E, Okamoto T, Higuchi T, Tatsumi K, Baba T, Murphy SK, Takakura K, Konishi I \& Fujii S 2009 Stress affects uterine receptivity through an ovarian-independent pathway. Human Reproduction 24 945-953. (https://doi.org/10.1093/humrep/den461)

Kupka MS, Ferraretti AP, de Mouzon J, Erb K, D'Hooghe T, Castilla JA, Calhaz-Jorge C, De Geyter C, Goossens V \& European IVF-Monitoring Consortium, for the European Society of Human Reproduction and Embryology 2014 Assisted reproductive technology in Europe, 2010: results generated from European registers by ESHRE. Human Reproduction 29 2099-2113. (https://doi. org/10.1093/humrep/deu175) https://raf.bioscientifica.com

https://doi.org/10.1530/RAF-21-0087 (c) 2022 The authors Published by Bioscientifica Ltd

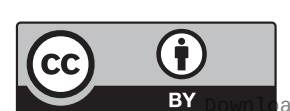

This work is licensed under a Creative Commons Attribution 4.0 International License. 
Ledee N, Petitbarat M, Chevrier L, Vitoux D, Vezmar K, Rahmati M, Dubanchet S, Gahery H, Bensussan A \& Chaouat G 2016 The uterine immune profile may help women with repeated unexplained embryo implantation failure after in vitro fertilization. American Journal of Reproductive Immunology 75 388-401. (https://doi.org/10.1111/aji.12483)

Martin MD, Danahy DB, Hartwig SM, Harty JT \& Badovinac VP 2017 Revealing the complexity in CD8 T cell responses to infection in inbred C57B/6 versus outbred Swiss mice. Frontiers in Immunology 8 1527. (https://doi.org/10.3389/fimmu.2017.01527)

Nakatsuka M, Habara T, Noguchi S, Konishi H \& Kudo T 2003 Impaired uterine arterial blood flow in pregnant women with recurrent pregnancy loss. Journal of Ultrasound in Medicine 22 27-31. (https://doi.org/10.7863/jum.2003.22.1.27)

Norwitz ER, Schust DJ \& Fisher SJ 2001 Implantation and the survival of early pregnancy. New England Journal of Medicine 345 1400-1408. (https://doi.org/10.1056/NEJMra000763)

Papageorghiou AT \& Leslie K 2007 Uterine artery Doppler in the prediction of adverse pregnancy outcome. Current Opinion in Obstetrics and Gynecology 19 103-109. (https://doi.org/10.1097/ GCO.0b013e32809bd964)

Patel JA, Patel AJ, Banker JM, Shah SI \& Banker MR 2019 Personalized embryo transfer helps in improving in vitro fertilization/ ICSI outcomes in patients with recurrent implantation failure. Journal of Human Reproductive Sciences 12 59-66. (https://doi.org/10.4103/jhrs. JHRS_74_18)

Rhee JS, Saben JL, Mayer AL, Schulte MB, Asghar Z, Stephens C, Chi MM \& Moley KH 2016 Diet-induced obesity impairs endometrial stromal cell decidualization: a potential role for impaired autophagy. Human Reproduction 31 1315-1326. (https://doi.org/10.1093/humrep/ dew048)

Richter KS, Shipley SK, McVearry I, Tucker MJ \& Widra EA 2006 Cryopreserved embryo transfers suggest that endometrial receptivity may contribute to reduced success rates of later developing embryos. Fertility and Sterility 86 862-866. (https://doi.org/10.1016/j. fertnstert.2006.02.114)

Ridder A, Giorgione V, Khalil A \& Thilaganathan B 2019 Preeclampsia: the relationship between uterine artery blood flow and trophoblast function. International Journal of Molecular Sciences 20 3263. (https://doi.org/10.3390/ijms20133263)

Ruiz-Alonso M, Blesa D, Diaz-Gimeno P, Gomez E, FernandezSanchez M, Carranza F, Carrera J, Vilella F, Pellicer A \& Simon C 2013 The endometrial receptivity array for diagnosis and personalized embryo transfer as a treatment for patients with repeated implantation failure. Fertility and Sterility 100 818-824. (https://doi. org/10.1016/j.fertnstert.2013.05.004)

Ruiz-Alonso M, Galindo N, Pellicer A \& Simon C 2014 What a difference two days make: 'personalized' embryo transfer (pET) paradigm: a case report and pilot study. Human Reproduction 29 1244-1247. (https://doi.org/10.1093/humrep/deu070)

Schjenken JE \& Robertson SA 2015 Seminal fluid signalling in the female reproductive tract: implications for reproductive success and offspring health. Advances in Experimental Medicine and Biology $\mathbf{8 6 8}$ 127-158. (https://doi.org/10.1007/978-3-319-18881-2_6)

Schjenken JE \& Robertson SA 2020 The female response to seminal fluid. Physiological Reviews 100 1077-1117. (https://doi.org/10.1152/ physrev.00013.2018)

Sudour H, Chastagner P, Claude L, Desandes E, Klein M, Carrie C \& Bernier V 2010 Fertility and pregnancy outcome after abdominal irradiation that included or excluded the pelvis in childhood tumor survivors. International Journal of Radiation Oncology, Biology, Physics 76 867-873. (https://doi.org/10.1016/j.ijrobp.2009.04.012)

Weber EM, Algers B, Hultgren J \& Olsson IA 2013 Pup mortality in laboratory mice - infanticide or not? Acta Veterinaria Scandinavica $\mathbf{5 5}$ 83. (https://doi.org/10.1186/1751-0147-55-83)

Winship AL, Stringer JM, Liew SH \& Hutt KJ 2018 The importance of DNA repair for maintaining oocyte quality in response to anti-cancer treatments, environmental toxins and maternal ageing. Human Reproduction Update 24 119-134. (https://doi.org/10.1093/humupd/ dmy002)

Wu F, Chen X, Liu Y, Liang B, Xu H, Li TC \& Wang CC 2018 Decreased MUC1 in endometrium is an independent receptivity marker in recurrent implantation failure during implantation window. Reproductive Biology and Endocrinology 16 60. (https://doi.org/10.1186/ s12958-018-0379-1)

Yang W, Wu Z, Yu M, Peng X, Lu W, Feng W \& Kang X 2019 Characteristics of midluteal phase uterine artery hemodynamics in patients with recurrent pregnancy loss. Journal of Obstetrics and Gynaecology Research 45 1230-1235. (https://doi.org/10.1111/jog.13944)

Received in final form 11 December 2021

Accepted 17 January 2022

Accepted Manuscript published online 17 January 2022 https://raf.bioscientifica.com

https://doi.org/10.1530/RAF-21-0087 (c) 2022 The authors Published by Bioscientifica Ltd

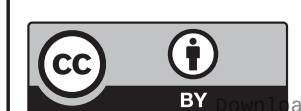

This work is licensed under a Creative Commons Attribution 4.0 International License. 